\title{
Intervenção fonoaudiológica e fisioterapêutica em uma mulher após acidente vascular cerebral isquêmico
}

\author{
Speech therapy and physiotherapy intervention in a woman after ischemic stroke
}

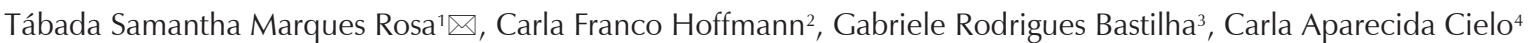 \\ ${ }^{1}$ Fisioterapeuta. Mestre em Distúrbios da Comunicação Humana, Universidade Federal de Santa Maria (UFSM), Santa Maria, RS. \\ 2 Fonoaudióloga. Mestre em Distúrbios da Comunicação Humana, UFSM. Santa Maria, RS. \\ ${ }^{3}$ Fonoaudióloga. Mestranda do Programa de Pós Graduação em Distúrbios da Comunicação Humana da UFSM. Santa Maria, RS. \\ ${ }^{4}$ Fonoaudióloga. Professor Associado do Departamento de Fonoaudiologia e do Programa de Pós-Graduação em Distúrbios da Comunicação Humana \\ UFSM. Santa Maria, RS.
}

\section{RESUMO}

Objetivos: Relatar um caso em que houve intervenção conjunta entre fonoaudiólogo e fisioterapeuta na reabilitação de uma paciente com acidente vascular cerebral isquêmico.

Descrição do caso: Paciente de 48 anos de idade, sexo feminino, acometida por acidente vascular cerebral do tipo isquêmico, há sete meses. Apresentou-se com disartrofonia e alterações do sistema estomatognático e sua funcionalidade, comprometimento sensorial, parestesia da mão direita, diminuição da coordenação motora grossa e fina em ambos os membros superiores, dificuldade na preensão palmar e fraqueza da musculatura intrínseca da mão direita, diminuição da consciência corporal e da propriocepção. Foi submetida a 12 sessões com a periodicidade de uma vez por semana de tratamento fonoterápico e fisioterapêutico. Após as intervenções a paciente apresentou aumento dos tempos máximos de fonação; melhora da coordenação pneumofonoarticulatória, da qualidade vocal e do foco de ressonância vertical; adequação e modulação da loudness; modulação do pitch; melhora da coordenação motora grossa e fina, da sensibilidade tátil e dolorosa, da oposição dos dedos, da preensão palmar, da consciência corporal, da propriocepção e da postura corporal global; e aumento da força muscular dos membros superiores.

Conclusões: A intervenção interdisciplinar da fonoaudiologia e fisioterapia proporcionou benefícios à paciente acometida por acidente vascular cerebral isquêmico, sendo que a mesma voltou a exercer sua atividade profissional e suas atividades de vida diária com efetividade e melhorou a comunicação com seus familiares.

DESCRITORES: voz; fonoaudiologia; fisioterapia; reabilitação; acidente vascular cerebral.

\section{ABSTRACT}

Aims: To report a case of joint intervention between speech therapist and physiotherapist in the rehabilitation of a patient with ischemic stroke. Case report: A 48-year-old female was affected by stroke of ischemic type seven months ago. The patient presented with dysarthrophonia and alterations of the stomatognathic system and its functionality, sensory impairment, right hand numbness, decreased gross and fine motor coordination of both upper limbs, difficulty in grip and weakness of the intrinsic muscles of the right hand, decreased proprioception and body awareness. She underwent 12 sessions of speech therapy and physical therapy with once a week frequency. After the sessions the patient showed increase of the maximum phonation time, improved pneumophonoarticulatory coordination, as well as improving in vocal quality and focus of vertical resonance; adequacy and modulation of loudness; pitch modulation; improved gross and fine motor coordination, tactile and pain sensitivity, opposition of the fingers, hand grip, body awareness, proprioception and overall body posture; and increased muscle strength of the upper limbs.

Conclusions: The interdisciplinary intervention with speech therapy and physical therapy provided benefits to this patient affected by stroke, allowing her to return to her full professional and daily activities, and improving communication with her family.

KEY WORDS: voice; speech, language and hearing sciences; physical therapy specialty; rehabilitation; stroke. 
Abreviaturas: AVC, acidente vascular cerebral; TMF, tempos máximos de fonação; MS, membro(s) superior(es); MI, membro(s) inferior(es).

\section{INTRODUÇÃO}

O acidente vascular cerebral (AVC) ocorre devido à lesão vascular e se apresenta como um déficit neurológico focal súbito. O AVC abarca lesões causadas por distúrbios da coagulação e hemodinâmicos, mesmo que não haja alterações detectáveis nas veias ou artérias [1]. De acordo com a etiologia do distúrbio que acomete a vascularização cerebral, o AVC pode ser classificado em isquêmico ou hemorrágico. Aproximadamente $80 \%$ dos casos devem-se à oclusão de uma artéria, seja por ateroma ou êmbolos secundários, caracterizando o AVC do tipo isquêmico [1,2]. No mundo ocidental, os casos de AVC constituem a segunda causa de morte em adultos e a primeira causa de incapacidade funcional, devido às sequelas associadas a déficits neurológicos e motores [3].

Danos neurológicos, como afasia, disartrofonia e apraxia, aliados à diminuição da função cognitiva, são os que mais interferem com a recuperação dos pacientes $[1,4]$. Os danos neurológicos relacionados à expressão oral eram, inicialmente, denominados de disartria e disartrofonia, termos considerados sinônimos. O termo disartria refere-se a uma dificuldade na articulação da fala em decorrência de um dano neurológico. Porém, estudos posteriores observaram que essa dificuldade não se limita apenas à fala, mas engloba também a respiração, a qualidade vocal, a frequência e a velocidade de fala. Dessa maneira, o termo disartrofonia foi considerado mais adequado para a descrição desses casos de dano neurológico $[5,6]$.

As sequelas motoras do AVC destacam-se por prejudicar a completa realização das atividades de vida diária e incluem diminuição da coordenação motora grossa e fina, fraqueza muscular, alteração da consciência corporal e proprioceptiva, entre outras [1,4]. Assim, é fundamental adotar condutas que possam reduzir as consequências do AVC [7]. Após o episódio de AVC, o tratamento de reabilitação deve ser iniciado imediatamente, de forma a amenizar as múltiplas incapacidades ocasionadas. Nesse contexto, a terapia multidisciplinar é necessária para o cuidado adequado do paciente, visando potencializar a reabilitação das sequelas $[1,5,8]$.

A reabilitação proporcionada pela ação conjunta da fonoterapia e da fisioterapia promove a capacidade de reorganização cerebral e melhora o comprometimento motor que afeta as atividades da vida diária e a comunicação. A participação do fonoaudiólogo e do fisioterapeuta na reabilitação do paciente pós-AVC constitui uma contribuição fundamental, que pode viabilizar maior resolução em relação aos efeitos da doença. No entanto, a literatura, principalmente sob uma perspectiva multidisciplinar, ainda é escassa no que se refere a estudos sobre a reabilitação fonoaudiológica e fisioterapêutica conjunta desses pacientes [8-10].

A partir do exposto, o objetivo deste estudo foi descrever o processo terapêutico e os resultados da intervenção conjunta da fonoaudiologia e da fisioterapia na reabilitação de uma paciente acometida por AVC do tipo isquêmico.

\section{RELATO DO CASO}

Esta pesquisa deriva de um projeto previamente aprovado pelo Comitê de Ética em Pesquisa da Universidade Federal de Santa Maria (no 23081.016945/ 2010-76), cujo Termo de Consentimento Livre e Esclarecido foi lido e assinado pela paciente conforme recomenda a resolução 466/12 do Conselho Nacional de Saúde, concordando que seus dados clínicos, com exceção dos de identificação, sejam utilizados para fins científicos.

Paciente de 48 anos de idade, sexo feminino, costureira, hipertensa e usuária de tabaco diariamente (antes e após o AVC). Não apresentava sinais prévios que indicassem a possibilidade de AVC e também não utilizava medicações que interferissem na função motora. A paciente foi acometida por AVC isquêmico aproximadamente sete meses antes do atendimento. Após o episódio agudo permaneceu com comprometimento do hemicorpo direito e disartrofonia, sendo encaminhada por um posto de saúde ao Serviço de Atendimento Fonoaudiológico (SAF), clínicaescola do curso de Fonoaudiologia da Universidade Federal de Santa Maria, localizada em Santa Maria/ $\mathrm{RS}$, local que proporciona à população atendimento multidisciplinar gratuito.

A paciente presentou-se com queixa de dificuldade em pegar objetos leves com a mão direita, os quais caiam, prejudicando o exercício de sua profissão, assim como dificuldades na comunicação oral. O exame otorrinolaringológico identificou laringe normal e a tomografia axial computadorizada mostrou hipodensidade parietal esquerda compatível com diagnóstico de AVC isquêmico. Na avaliação neurológica foi observado agnosia táctil dígito-manual direita com apraxia na mesma mão, moderada disartria e frequente falta de palavras na emissão de frases. 
$\mathrm{Na}$ avaliação miofuncional orofacial realizada pela fonoaudióloga, a paciente apresentou sensibilidade intraoral diminuída à esquerda; ângulo nasolabial maior do que $90^{\circ}$; lábio superior cobrindo mais que 2/3 dos incisivos superiores; lábios assimétricos à direita, com tensão e mobilidade diminuídas; músculo mentual e bochechas com tensão diminuída, sendo a bochecha assimétrica à esquerda; língua com tensão, sensibilidade e mobilidade diminuídas; prótese dentária superior mal adaptada, em consequência do emagrecimento de $10 \mathrm{~kg}$ após o AVC; e mastigação assimétrica à esquerda.

No exame fonoarticulatório, a paciente apresentou vibração de língua insuficiente para a produção do fonema $/ \mathrm{r} / \mathrm{has}$ palavras com encontros consonantais $\mathrm{com} / \mathrm{r} / \mathrm{em}$ onset inicial (pr, br, tr, dr, cr, gr, fr) e onset medial (vr). $\mathrm{Na}$ análise perceptivoauditiva da voz, apresentou pitch moderadamente grave; loudness moderadamente fraca; ressonância vertical moderadamente laringofaríngea e ressonância horizontal moderadamente posterior; modulação de pitch e de loudness restrita com condições de modular; voz moderadamente comprimida, pastosa, monótona e discretamente rouca, com entradas discretas em fry, hesitações constantes e dificuldade em nomear.

A velocidade de fala apresentou-se diminuída (fala espontânea $=47$ palavras $/$ minuto; leitura de texto $=65$ palavras/minuto), e a articulação era hipotensa e lenta. A dificuldade durante a leitura oral também poderia ser justificada pela baixa escolaridade da paciente ( $5^{\mathrm{a}}$ série do ensino fundamental). $\mathrm{O}$ modo respiratório em repouso foi nasal e, durante a fala, oronasal, estando ambas as formas adequadas. Os tempos máximos de fonação (TMF) das vogais apresentaram média de 10 segundos, enquanto que a contagem de números foi de nove números em 14,2 segundos. A comparação destes resultados indicou hiperfunção no mecanismo de fala [11].

A relação entre o tempo máximo de fonação obtido e o previsto foi de 0,7. O valor do Fluxo Aéreo Adaptado foi de $230 \mathrm{ml} / \mathrm{s}$ e o do Cociente Fônico Simples foi de $261,6 \mathrm{ml} / \mathrm{s}$. Todos esses valores se mostraram inadequados, tendo como referência a normalidade para mulheres adultas, sugerindo escape aéreo na fonação [11]. O valor da relação entre as vogais /e/ áfono ([e்]) e sonoro ([e]) e entre os fricativos [s] e [z] (ambos de 0,6) indicaram discreta hipercontração na fonação, provavelmente compensatória ao escape aéreo [11]. O valor do Cociente Fônico Composto de $225,4 \mathrm{ml} / \mathrm{s}$ esteve dentro da faixa da normalidade [11], não podendo ser interpretado isoladamente frente aos demais achados da avaliação. Tais resultados evidenciam falta de coordenação entre as forças aerodinâmicas da corrente pulmonar e mioelásticas da laringe durante a fonação.

Com base nas avaliações, a paciente recebeu o diagnóstico fonoaudiológico de disartrofonia e alterações do sistema estomatognático e suas funções, decorrentes do AVC isquêmico. Elaborou-se o planejamento fonoterapêutico cujos objetivos foram: melhorar a articulação da fala; aumentar os TMF; desenvolver o controle da descontração progressiva da musculatura respiratória; desenvolver o apoio respiratório abdominal; aumentar a resistência vocal; melhorar o foco de ressonância; melhorar a qualidade vocal; melhorar a prosódia; estimular a plasticidade vocal; aprimorara funcionalidade das estruturas do sistema estomatognático; melhorar a coordenação pneumofonoarticulatória, o pitch e a loudness; e aperfeiçoar a velocidade de fala. Além disso, a paciente foi encaminhada para tratamento odontológico para adequação da prótese dentária.

$\mathrm{Na}$ avaliação fisioterapêutica, constatou-se parestesia de membro superior (MS) direito; diminuição da coordenação motora grossa e fina em ambos os MS; dificuldade de preensão palmar da mão direita; fraqueza da musculatura intrínseca da mão direita apresentando grau 2 de força muscular; diminuição da consciência corporal e proprioceptiva; tipo respiratório misto (uso da musculatura acessória e abdominal); comprometimento sensorial, com sensibilidade térmica preservada somente no lado acometido; e amplitude de movimento normal de MS. A paciente não apresentava déficit de equilíbrio estático ou dinâmico, não utilizava medicações que interferissem na função motora e não apresentava desajustes posturais ou dissociação de cinturas na deambulação, não necessitando de dispositivos auxiliares para a marcha. Em relação às alterações posturais decorrentes do $\mathrm{AVC}$, foram identificados cabeça anteriorizada, protrusão de ombros, elevação escapular, anteversão pélvica, joelhos valgos e pés pronados. Importante ressaltar ainda que a paciente apresentou discreta alteração da capacidade funcional, sendo capaz de se autocuidar, diagnóstico confirmado através da Escala de Barthel.

Com base no resultado das avaliações, a paciente recebeu o diagnóstico fisioterapêutico de hemiparesia de membro superior direito decorrente de AVC isquêmico, e elaborou-se planejamento fisioterapêutico cujos objetivos foram: melhora da coordenação motora grossa e fina; aumento da preensão palmar direita; promoção da adequação do tipo respiratório (costodiafragmático-abdominal) e de uma melhora da postura corporal; estímulo da consciência e da propriocepção 
corporal; e estímulo da sensibilidade dolorosa e tátil, procurando aumentar a funcionalidade do hemicorpo afetado nas atividades da vida diária, proporcionando autonomia e, consequentemente, buscando uma melhor qualidade de vida.

A paciente foi submetida a 12 sessões de atendimento fonoaudiológico e fisioterapêutico conjuntos, com duração de uma hora e com frequência semanal. Inicialmente a fisioterapeuta realizava atividades para aquecimento corporal e, após, a fonoaudióloga realizava aquecimento e exercícios vocais. $\mathrm{Na}$ sequência, eram passados exercícios de fisioterapia, finalizando com desaquecimento corporal e vocal.

No âmbito fonoaudiológico, as atividades realizadas foram: execução de sons de apoio fricativos sonoros e basais; exteriorização lingual; exercícios cervicais sonorizados; rotação de língua no vestíbulo oral; modulação de pitch e loudness; exercícios com escalas musicais; monitoração visual, auditiva e cinestésica; sobrearticulação; exercícios com imagens mentais; estalo, protrusão, retração e lateralização de língua; varredura de palato com a língua; inflação das bochechas; exercício labial de bico-sorriso; e mastigação bilateral com diferentes consistências. As atividades eram realizadas em frente ao espelho, com orientações sobre o tipo respiratório costodiafragmático-abdominal. Também eram efetuadas leituras de notícias para melhora de vocabulário e treino com diferentes entonações para promover a prosódia.

No decorrer dos atendimentos fisioterapêuticos, foram utilizados diversos recursos, como tecidos, balões, bastões, toalhas, faixas elásticas, cadeiras, mesas e todo o espaço físico possível de ser explorado para promover maior funcionalidade nas sessões. Estimulou-se a sensibilização do lado afetado com materiais de diferentes texturas e formas (esponja, pincel, caneta de ponta grossa e fina, algodão, agulha), tendo sido aplicadas diferentes pressões em diversos lugares do corpo. Além disso, realizaram-se tarefas que estimulassem a coordenação motora, como exercícios índex-nariz-orelha, movimentos alternados de MS, e treinamento do movimento fino das mãos, com atividades como levar colher de água à boca, transferir pequenos objetos de um lugar para outro, catar grãos, escrever frases, passar linha na agulha e utilizar pinça.

Também foram feitos exercícios para a musculatura intrínseca da mão, nos quais a paciente era solicitada a apoiar a mão em uma toalha de rosto sobre a mesa e, logo após, flexionar e estender os dedos, de modo que enrugasse e esticasse a toalha; bem como segurar uma bola média (do tamanho da palma da mão) com a mão direita, devendo apertá-la o máximo que conseguisse e permanecer assim durante dez segundos.

Foram realizados, ainda, exercícios para a sensibilização do pé direito, passando-se uma toalha de rosto em toda a superfície e, à medida que a paciente referia perceber a sensação, substituía-se a toalha de rosto por tecidos mais finos, como malha de algodão, viscose e seda. Na sequência, foram efetuados exercícios com os MS e membros inferiores (MI) em frente ao espelho, para a propriocepção e conscientização corporal com auxílio de balões, bastões e técnica de Facilitação Neuromuscular Proprioceptiva, visando à melhora da percepção corporal no espaço; mobilizações articulares em nível de ombros, escápulas, punhos, mãos, quadris, joelhos e pés; bem como alongamentos globais de MS e MI com uso de faixa elástica. Por fim, executaram-se relaxamento da musculatura acessória e treinamento respiratório costodiafragmático-abdominal em tempos, associados aos MS. Durante o estudo, a paciente não foi submetida a quaisquer outras modalidades de terapia voltadas para a reabilitação do MS.

Após treinamento em sessão, a paciente foi orientada a praticar em casa os exercícios abordados na terapia fonoaudiológica com o tipo respiratório correto, monitorando a postura em frente ao espelho. Foram recomendados três exercícios vocais e dois exercícios miofuncionais com três séries de 15 repetições cada, com intervalos de descanso entre as séries. Ainda, recebeu da fisioterapeuta a indicação de realizar os seguintes exercícios em casa, em três séries com dez repetições cada: segurar uma bola média com a palma da mão, apertando-a fortemente o máximo que conseguisse, mantendo essa força por dez segundos; logo após, em uma toalha de rosto, fazer movimentos para enrugá-la com os dedos da mão direita e depois esticá-la (ambos os exercícios indicados para o fortalecimento da musculatura intrínseca da mão); além de fazer movimentos de pinça com os dedos indicador e polegar, como catar feijão ou arroz, para aprimorar a capacidade motora fina. A paciente também foi orientada que fizesse alongamentos globais de MS e MI ao acordar pela manhã; evitasse carregar objetos pesados; flexionasse os joelhos ao pegar objetos do chão; e mantivesse toda a coluna sempre apoiada a um encosto ao sentar. Todas as orientações visaram à melhora da postura, no intuito de diminuir e/ou prevenir contraturas e encurtamentos.

Como resultado do tratamento, após as 12 sessões de terapia fonoaudiológica e fisioterapêutica realizadas em conjunto, a paciente foi reavaliada. Na reavaliação neurológica, o laudo foi de agnosia tátil dígito-manual 
direita com apraxia na mesma mão, discreta disartria (anteriormente moderada) e eventual (anteriormente frequente) falta de palavras na emissão de frases.

Houve melhora na realização das praxias de lábios e na simetria das comissuras labiais; a paciente conseguiu articular todas as palavras, incluindo aquelas nas quais apresentou dificuldade no início do tratamento. A voz apresentou-se discretamente pastosa e comprimida, com pitch moderadamente grave, loudness e modulação de pitch e de loudness adequadas, foco vertical de ressonância discretamente laringofaríngeo e foco horizontal de ressonância moderadamente posterior.

Também ocorreu aumento da média dos TMF das vogais $(14,4 \mathrm{~s})$ e dos números contados por segundo (16 números em 12s), indicando maior quantidade de fala encadeada em menor intervalo de tempo; aumento da velocidade de fala espontânea para 96 palavras/minuto e da velocidade de fala durante a leitura de texto para 76 palavras/minuto. Verificou-se, ainda, melhora dos valores de Cociente Fônico Composto $(205,2 \mathrm{ml} / \mathrm{s})$, Cociente Fônico Simples $(153,8 \mathrm{ml} / \mathrm{s})$, Fluxo Aéreo Adaptado $(155,5 \mathrm{ml} / \mathrm{s})$, relação s/z $(0,9)$ e relação entre o tempo máximo de fonação obtido e o previsto $(0,9)$. A paciente referiu, ao término dos atendimentos, ter melhorado a comunicação com familiares.

Quanto ao processo fisioterapêutico, houve melhora significativa da coordenação motora grossa e fina, observada por meio da efetividade na execução de atividades funcionais; melhora da sensibilidade tátil e dolorosa de MS e MI, da oposição dos dedos, da preensão palmar direita, da consciência, da propriocepção e da postura corporal. Também houve desenvolvimento do tipo respiratório costo-diafragmático-abdominal, promovendo maior controle respiratório; e aumento da força muscular de MS, apresentando grau cinco. Além disso, a paciente referiu ter voltado a executar a costura, sua atividade profissional, e não mais deixar cair objetos leves da mão direita.

\section{DISCUSSÃO}

Estudo recente realizado com 123 pacientes durante internação hospitalar após AVC verificou que aproximadamente 37 minutos de terapia diária de fisioterapia e terapia ocupacional e 13 minutos de terapia fonoaudiológica ocasionaram ganhos significativos na recuperação funcional dos pacientes, inclusive nas áreas motora e cognitiva [11] embora esse tempo de terapia tenha sido inferior ao que é sugerido pela "The Canadian Best Practice Recommendations for Stroke Care" (2008), de uma hora por dia por cada terapeuta. O presente estudo diferencia-se do citado devido ao fato da paciente não ter sido atendida durante internação hospitalar, e sim em uma clínica ambulatorial, sete meses após o episódio de AVC, recebendo uma hora de atendimento semanal durante 12 semanas. $\mathrm{O}$ atendimento descrito neste relato mostra que essa paciente, assim como aqueles atendidos durante a hospitalização, também apresentou melhora nos aspectos motores e de comunicação.

Em outro estudo, 14 pacientes foram avaliados durante internação hospitalar após ocorrência de AVC, com intervalo de duas semanas entre as avaliações. Os níveis de atividade física, cognitiva e social dos pacientes permaneceram baixos nas duas semanas, mesmo com a melhora do nível de independência e humor. Estes resultados sugerem a necessidade de explorar estratégias para estimular atividades dentro de ambientes de reabilitação, considerando as que já fazem parte da rotina do paciente [3].

Ainda não há consenso ou uma relação entre tempo de terapia e ganho funcional do paciente após AVC, por isso são necessários novos estudos visando a fornecer evidências científicas e melhorar o cuidado do paciente.

A fisioterapia e a fonoaudiologia fazem parte da reabilitação do paciente pós $\mathrm{AVC}$, sendo que a recuperação do indivíduo depende de fatores intrínsecos, como idade do paciente, local do sistema nervoso central lesionado e histórico de saúde, bem como de fatores extrínsecos, como aspectos ambientais e psicossociais e momento da intervenção terapêutica $[9,11]$. No que tange aos aspectos intrínsecos, o tipo isquêmico, encontrado em $90 \%$ dos casos de AVC, tem prognóstico de recuperação mais favorável quando comparado ao AVC hemorrágico $[1,10]$. Sob essa perspectiva, a recuperação dessa paciente pode ter sido facilitada pelo mecanismo de lesão, respondendo de forma muito efetiva à estimulação fonoaudiológica e fisioterapêutica [1,2]. O histórico médico de hipertensão arterial da paciente é compatível com a ocorrência de AVC. A hipertensão arterial é um fator de risco predominante, estando presente em $90 \%$ dos pacientes com AVC [1,12].

As deficiências sensoriais mais frequentes como consequências de AVC estão relacionadas com a sensibilidade tátil e dolorosa, e podem ocorrer caso algum nervo sensorial seja afetado pelo rompimento das terminações nervosas. A perda do tato superficial e do tato da dor é comum, podendo ocorrer disfunção perceptiva geral e risco de autolesões. Na prática clínica fisioterapêutica são utilizados exercícios para sensibilização do membro afetado, visando à 
recuperação da sensibilidade tátil e, por conseguinte, auxiliando a realização das atividades motoras, pois a presença do feedback tátil melhora a consciência corporal e apercepção do movimento, reduzindo ainda o risco de acidentes $[13,14]$.

A coordenação motora resulta da integridade neuromuscular, de forma que as lesões causadas pelo AVC provocam diminuição das habilidades motoras amplas e finas, ocasionando incapacidade para desempenhar as atividades diárias e outras tarefas que requeiram preensão de objetos [15]. No presente estudo, verificou-se melhora gradativa do desempenho da preensão palmar no decorrer das sessões, com isso facilitando a realização de funções como segurar objetos pequenos. Cabe destacar que a recuperação dessas funções foi extremamente importante para a execução da profissão de costureira da paciente.

No âmbito fonoaudiológico, a disartrofonia, consequência do comprometimento neurológico, devido à sua íntima relação com a fala, é considerada pelos pacientes acometidos pelo distúrbio e por seus familiares como o principal fator de isolamento social e de depressão [6,8]. Devido ao comprometimento da coordenação muscular, a imprecisão articulatória é a alteração mais frequente nos casos de disartrofonia [4]. No que se refere aos fonemas, a produção das consoantes é mais severamente afetada quando comparada à das vogais, como observado no exame articulatório da paciente deste caso [15]. Corroborando com o que foi encontrado neste estudo, a velocidade de fala diminuída foi relatada em um estudo sobre a velocidade de fala espontânea e de leitura oral em pacientes disártricos quando comparados com pacientes sem queixas articulatórias, como no caso da paciente estudada [15].

Nos casos de dano neurológico, conforme a localização da lesão, os nervos responsáveis pelos músculos respiratórios podem estar enfraquecidos, de modo que a capacidade vital fica diminuída [12]. Isso ocasiona produção vocal com frases mais curtas e loudness fraca, como no caso apresentado [11]. Esta paciente, no início do tratamento, apresentava loudness moderadamente fraca e, na reavaliação, loudness normal. A loudness alterada pode interferir negativamente na comunicação funcional do indivíduo $[4,6]$.

Estudo recente avaliou a voz, por meio da avaliação perceptivo-auditiva, de 10 idosos acometidos por AVC, e verificou a presença predominante das características vocais de rugosidade, soprosidade, instabilidade e pastosidade, além de alteração no controle motor laríngeo, constatado pela velocidade reduzida de fala [16]. Tais características assemelham-se às encontradas no caso presente, também tendo apresentado melhora após o processo terapêutico.

A localização do AVC pode comprometer, também, a inervação dos músculos adutores das pregas vocais, gerando fechamento glótico incompleto com qualidade vocal soprosa ou hiperadução com voz comprimida, além de dificuldades na variação de pitch e de loudness durante o discurso [12]. O laudo otorrinolaringológico desta paciente foi normal, mas, no início do tratamento, ela apresentava voz moderadamente comprimida, provavelmente devido ao esforço compensatório empregado para "melhorar" a fala. Ao final do tratamento, o grau de alteração foi discreto, melhorando a efetividade da comunicação.

Ressalta-se, ainda, que a adequação da força e do controle muscular respiratório, da descontração progressiva dessa musculatura e do tipo respiratório costo-diafragmático-abdominal, realizada de maneira conjunta entre as terapeutas, possibilitou o aumento da resistência vocal, da coordenação pneumofonoarticulatória e da loudness da paciente [11,17].

O sistema nervoso central possui a capacidade de modificar-se continuamente, sendo a plasticidade uma consequência obrigatória de cada input sensorial e de cada atividade motora [7]. Pôde-se comprovar essa teoria por meio do caso exposto, em que a intervenção, a adesão da paciente à terapia, o tipo de lesão e a neuroplasticidade foram fundamentais para a melhora dos aspectos afetados, permitindo à paciente voltar às atividades profissionais e sociais, saindo do isolamento que tanto afeta os indivíduos acometidos por esse tipo de enfermidade.

Revisão sistemática de literatura analisou 14 estudos na forma de ensaios clínicos randomizados com foco em intervenções multidisciplinares para pacientes com AVC que viviam em casa após hospitalização. Nenhum dos estudos mostrou efeitos favoráveis da intervenção multidisciplinar nas atividades da vida diária, dois estudos relataram efeitos favoráveis em termos de qualidade de vida e nenhum avaliou a participação social dos indivíduos [4]. Há pouca evidência para a eficácia da assistência multidisciplinar em pacientes com AVC após alta hospitalar, sugerindo a realização de pesquisas que forneçam mais informações sobre os resultados da assistência multidisciplinar e considerem a autopercepção do paciente, como é o caso do presente relato. Espera-se que relatos como este estimulem a realização de pesquisas com amostras grandes e presença de grupos de estudo e de controle, para a correta avaliação da intervenção multidisciplinar nos casos de AVC. 
Em relação ao caso dessa mulher com sequelas de AVC isquêmico, pode-se concluir que intervenção multidisciplinar fonoaudiológica e fisioterapêutica foi efetiva, pois a paciente apresentou melhora relevante após 12 sessões semanais de intervenção conjunta, recuperando-se da disartrofonia e readquirindo as capacidades motoras, podendo assim retornar à sua atividade profissional e às atividades da vida diária, além de reaver a capacidade de comunicação interpessoal.

\section{REFERÊNCIAS}

1. Cruz KCT, Diogo MJDE. Avaliação da capacidade funcional de idosos com acidente vascular encefálico. Acta Paul Enferm. 2009;22(5): 666-72. http://dx.doi.org/10.1590/S0103-21002009000500011

2. Appelros P, Stegmayr B, Terent A. Sex Differences in Stroke Epidemiology: A Systematic Review. Stroke. 2009;40(1):1082-90. http:// dx.doi.org/10.1161/STROKEAHA.108.540781

3. Janssen H, Ada L, Bernhardt J, McElduff P, Pollack M, Nilsson M, Spratt N. Physical, cognitive and social activity levels of stroke patients undergoing rehabilitation within a mixed rehabilitation unit. Clinical Rehabilitation. 2014;28(1):91-101. http://dx.doi. org/10.1177/0269215512466252

4. Fens M, Vluggen TP, Haastregt JV, Verbunt JA, Beusmans GH, Heugten CMV. Multidisciplinary care for stroke pa tients living in the community: A systematic review. J Rehabil Med. 2013;45(4):321-30. http://dx.doi.org/10.2340/16501977-1128

5. Santos MD, Mac-Kay APMG. Resultados preliminares do estudo da compreensão em sujeitos disártricos. Rev CEFAC. 2011;13(5): 825-31. http://dx.doi.org/10.1590/S1516-18462011005000042

6. Bowen A, Hesketh A, Patchick E, Young A, Davies L, Vail A, Long A, Watkins C, Wilinson M, Pearl G, Ralph M, Tyrrell P. Effectiveness of enhanced communication therapy in the first four months after stroke for aphasia and dysarthria: a randomised controlled trial. BMJ. 2012;345:e4407. http://dx.doi.org/10.1136/bmj.e4407

7. Garcia LT, Lefèvre AP. Os efeitos do canto coral na disartrofonia. In: IX Jornada de Iniciação Científica. Universidade Guarulhos: São Paulo; 2011. p. 42.

8. Pereira ABCNG, Alvarenga H, Pereira Júnior RS, Barbosa MTS. Prevalência de acidente vascular cerebral em idosos no Município de Vassouras através do rastreamento de dados do Programa Saúde da Família. Cad Saúde Pública. 2009;25(9):1929-36. http://dx.doi. org/10.1590/S0102-311X2009000900007

9. Arthur AM, Vanini TM, Lima NM, Iano Y, Arthur R. Tratamentos fisioterapêuticos em pacientes pós-AVC: uma revisão do papel da neuroimagem no estudo da plasticidade neural. Ensaios e C. 2010;14(1):187-208.

10. Godoy JF, Brasolotto AG, Berretin-Félix G, Fernandes Adriano Y. Neuroradiology and voice findings in stroke. CoDAS. 2014;26(2): 168-74. http://dx.doi.org/10.1590/2317-1782/2014531IN

11. Foley N, McClure A, Meyer M, Salter K, Bureau Y, Teasell R. Inpatient rehabilitation following stroke: amount of therapy received and associations with functional recovery. Disability \& Rehabilitation. 2012;34(25):2132-38. http://dx.doi.org/10.3109/09638288.2012.676145

12. Ribeiro AF, Ortiz KZ. Perfil populacional de pacientes com disartria atendidos em hospital terciário. Rev Soc Bras Fonoaudiol 2009;14(4):446-53. http://dx.doi.org/10.1590/S1516-80342009000400004

13. Talarico TR, Venegas MJ, Ortiz KZ. Perfil populacional de pacientes com distúrbios da comunicação humana decorrentes de lesão cerebral, assistidos em hospital terciário. Rev CEFAC. 2011;13(2):330-9. http://dx.doi.org/10.1590/S1516-18462010005000097

14. Behlau M. Voz o Livro do Especialista. 2⿳a ed. Rio de Janeiro: Revinter; 2013. 568p.

15. Costa FA, Silva DLA, Rocha, VM. Severidade clínica e funcionalidade de pacientes hemiplégicos pós-AVC agudo atendidos nos serviços públicos de fisioterapia de Natal (RN). Ciênc Saúde Colet. 2011;16(1):1341-8. http://dx.doi.org/10.1590/S1413-81232011000700068

16. Nunes MCA, Jurkiewicz AL, Santos RS, Furkim AM, Massi G, Pinto GS, Lange MC. Correlação entre a lesão encefálica e a disfagia em pacientes adultos com acidente vascular encefálico. Int Arch Otorhinolaryngol. 2012;16(3):313-21. http://dx.doi.org/10.7162/S180997772012000300003

17. Langhorne P, Bernhardt J, Kwakkel G. Stroke rehabilitation. Lancet. 2011;377(9778):1693-702. http://dx.doi.org/10.1016/S01406736(11)60325-5 $C$ 